九州大学学術情報リポジトリ

Kyushu University Institutional Repository

\title{
SOME NONPARAMETRIC ESTIMATORS OF A LOCATION PARAMETER
}

Yanagawa, Takashi

Osaka University

https://doi.org/10.5109/13022

出版情報: 統計数理研究. 12 (3/4), pp.11-19, 1967-03. Research Association of Statistical Sciences

バージョン :

権利関係 : 


\title{
SOME NONPARAMETRIC ESTIMATORS OF A LOCATION PARAMETER
}

\author{
By \\ Takashi Yanagawa \\ (Received December 1, 1966)
}

\section{$\S 1$. Introduction and Summary.}

The purpose of this paper is to discuss some nonparametric estimators of a location parameter, especially their asymptotic relative efficiencies relative to the sample mean.

Let $X_{1}, X_{2}, \cdots, X_{n}$ be a random sample from the population with cumulative distribution function $F(x-\theta)$, where $\theta$ is a location parameter and $F(x)$ is assumed to belong to the family $\mathfrak{F}$ of all distribution functions that are symmetric about the origin and absolutely continuous with respect to the Lebesgue measure. Let $\hat{\theta}_{p}$ be the median of the means of all p-tuple $\left(X_{i_{1}}, X_{i_{2}}, \cdots, X_{i p}\right),\left(\begin{array}{c}N \\ p\end{array}\right)$ in number, drawn from $X_{1}, X_{2}, \cdots, X_{N}$, i.e.

$$
\hat{\theta}_{p}=\underset{i_{1}<i_{2}<\cdots<i p}{M e d} \underset{i_{i 1}+X_{i_{2}}+\cdots+X_{i p}}{p},
$$

which we shall propose as an estimator of $\theta$.

In the simplest case $p=1, \hat{\theta}_{1}$ is the sample median. In a recent paper [2] J. L. Hodges and E. L. Lehmann derived the estimator $\hat{\theta}_{2}$ of $\theta$ from the one sample Wilcoxon statistic. Some of their results are as follows. The asymptotic efficiency of $\hat{\theta}_{1}$ relative to the sample mean $\bar{X}$, denoted A.R.E. $\left(\hat{\theta}_{1} \mid \bar{X}\right)$, in the sence of reciprocal ratio of asymptotic variances, is $4 \sigma_{f}^{2} f_{(0)}^{2}$, where $f$ denotes the density corresponding to $F$ and $\sigma_{f}^{2}$ its variance, while A.R.E. $\left(\hat{\theta}_{2}(\bar{X})=12 \sigma_{f}^{2}\left(\int f_{(x)}^{2} d x\right)^{2}\right.$. The infimum of these efficiencies with respect to the underlying distribution are well known to be 0 and 0.864 , respectively. Our investigation is a generalization of these results.

In Section 2 we shall discuss some properties of $\hat{\theta}_{p}$. In Section 3 we shall state our main results that the infimum of A.R.E. $\left(\hat{\theta}_{p}^{\prime} X\right)$ with respect to the population distribution is always greater than or equal to 0.864 for even $p$, but not so for odd $p$, even if $p \geq 3$. In Section 4 we shall consider the case in which $N$ observations are divided into $p$ groups and define alternative estimators of $\theta$ and recomend some of them as estimators of $\theta$.

\section{§2. Some properties of $\hat{\theta}_{p}$.}

By means of a rank test statistic $T(x), X=\left(X_{1}, \cdots, X_{N}\right)$, which satisfies the condition (1) $T(x+a)$ is a nondecreasing function of $a$ for all $x$, (2) 
$E_{0} T(x)=\mu$, where $\mu$ is independent of $F$ and $E_{0}$ denotes the expectation under $\theta=0$, Hodges and Lehmann [2] defined the estimator of $\theta$ as follows.

$$
\hat{\theta}=\frac{\theta^{*}+\theta^{* *}}{2},
$$

where $\theta^{*}=\inf \{\theta ; T(x-\theta)<\mu\}$ and $\theta^{* *}=\sup \{\theta ; T(x-\theta)>\mu\}$.

If we put

$$
T(X)=\frac{1}{\left(\begin{array}{c}
N \\
p
\end{array}\right)} \Psi\left\{\left(i_{1} \cdots i_{p}\right) ; X_{i_{1}}+\cdots+X_{i_{p}}>0, i_{1}<i_{2}<\cdots<i_{p}\right\},
$$

where means the number of p-tuble $\left(i_{1} i_{2} \cdots i_{p}\right)$ such that $X_{i_{1}}+X_{i_{2}}+\cdots+X_{i_{p}}$ $>0$, then the estimator $\hat{\theta}_{p}$ and $\hat{\theta}$ defined in (1.1) and (2.1), respectively, are seen to be identical. Therefore all results in [2] hold for the estimator $\hat{\theta}_{p}$, i.e. (a) the distribution of $\hat{\theta}_{p}$ is absolutely continuous with respect to the Lebesgue measure, (b) the distribution of $\hat{\theta}_{p}$ is symmetric about $\theta$, so that $\hat{\theta}_{p}$ is an unbiased estimator of $\theta$, (c) $\hat{\theta}_{p}$ is translation invariant, (d) the asymptotic relative efficiency of the test based on the test statistic $T(x)$ defined in (2.2) with respect to t-test is equal to A.R.E. $\left(\hat{\theta}_{p} \mid \bar{X}\right)$, (e) we shall have the lemma below (see [2] p. 607).

Lemma 2.1. For $T(X)$ and $\hat{\theta}_{p}$ defined by (2. 2) and (2. 1), respectively, and for all a

$$
P\{T(X-a)<\mu\} \leq P\left\{\hat{\theta}_{p} \leq a\right\} \leq P\{T(X-a) \leq \mu\} .
$$

Let

$$
G_{p}(y)=\int \cdots \int F\left(y-x_{2}-\cdots-x_{p}\right) f\left(x_{2}\right) \cdots f\left(x_{p}\right) d x_{2} \cdots d x_{p},
$$

$$
\lambda_{p}(F)=\int f(x) G_{p-1}^{2}(\theta) d x,
$$

and let $g_{p}(y)$ be the p.d.f. of $G_{p}(y)$. Then we obtain the following theorem.

Theorem 2.1. Suppose $G_{p}(y)$ has the derivative $g_{p}(o)=0$ at $y=0$. Then $N^{1 / 2}\left(\hat{\theta}_{p}-\theta\right)$ has a limiting normal distribution with mean $O$ and variance $\left(\lambda_{p}(F)-1 / 4\right) / g_{p}^{2}(o)$.

Proof For any real $u$, let

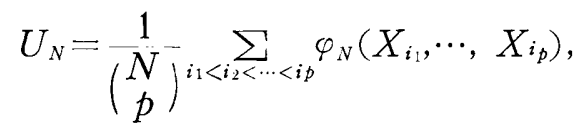

where $\varphi_{N}\left(x_{1}, \cdots, x_{p}\right)=1$ if $x_{1}+\cdots+x_{p}>p u / N^{1 / 2},=0$ otherwise. Note that $\mu=$ $E_{0} T(X)=1 / 2$ and $T\left(X-u / N^{1 / 2}\right)=U_{N}$, then from above (c) and Lemma 2. 1

$$
\lim _{N \rightarrow \infty} P_{\theta}\left\{N^{1 / 2}\left(\hat{\theta}_{p}-\theta\right) \leq u\right\}=\lim _{N \rightarrow \infty} P_{0}\left\{\hat{\theta}_{p} \leq u / N^{1 / 2}\right\}
$$




$$
\begin{aligned}
& =\lim _{N \rightarrow \infty} P_{0}\left\{T\left(X-u / N^{1 / 2}\right) \leq \frac{1}{2}\right\} \\
& =\lim _{N \rightarrow \infty} P_{0}\left\{N^{1 / 2}\left(U_{N}-E_{0} U_{N}\right) \leq N^{1 / 2}\left(1 / 2-E_{0} U_{N}\right)\right\} .
\end{aligned}
$$

Since $U_{N}$ is a $U$-statistic, for which $\varphi_{N}$ is uniformly bounded, it follows from the general theory of $U$-statistic [3] that $N^{1.2}\left(U_{N}-E_{0} U_{N}\right)$ has a limiting normal distribution with mean 0 and variance $p^{2}\left[P_{0}\left\{X_{1}+X_{2}+\cdots+\right.\right.$ $\left.\left.X_{p}>0, X_{1}+X_{2}^{\prime}++X_{p}^{\prime}>0\right\}-\left(P_{0}\left\{X_{1}+\cdots+X_{p}>0\right\}\right)^{2}\right]=p^{2}\left(\lambda_{p}(F)-1 / 4\right)$, where the $X_{1}^{\prime}$ and $X_{j}$ are independent and identically distributed with c.d.f. $F(x)$. On the other hand $N^{1 / 2}\left(1 / 2-E_{0} U_{N}\right)=N^{1 / 2}\left(G_{p}\left(p u / N^{1 / 2}\right)-1 / 2\right)=N^{1 / 2}\left(G_{p}(p u)\right.$ $\left.\left.N^{1 / 2}\right)-G_{p}(0)\right) \rightarrow p u g_{p}(0)$, as $N \rightarrow \infty$, which completes the proof.

\section{$\S 3$. Asymptotic efficiency of $\hat{\theta}_{p}$}

It is well known that $N^{1 / 2}(X-\theta)$ has a limiting normal distribution with mean 0 and variance $\sigma_{f \cdot}^{2}$ Therefore from Theorem 2. 1

$$
\begin{aligned}
& \text { A.R.E. }\left(\hat{\theta}_{p} \bar{X}\right)=\sigma_{f}^{2} g_{p}^{2}(0) /\left(\lambda_{p}(F)-\frac{1}{4}\right), \\
& \text { A.R.E. }\left(\hat{\theta}_{p} \hat{\theta}_{q}\right)=g_{p}^{2}(0)\left(\lambda_{q}(F)-\frac{1}{4}\right) / g_{q}^{2}(0)\left(\lambda_{p}(F)-\frac{1}{4}\right) .
\end{aligned}
$$

Especially

$$
\begin{aligned}
& \text { A.R.E. }\left(\hat{\theta}_{p} \hat{\theta}_{1}\right)=g_{p}^{2}(0) / 4 f^{2}(0)\left(\lambda_{p}(F)-\frac{1}{4}\right), \\
& \text { A.R.E. }\left(\hat{\theta}_{p} \hat{\theta}_{2}\right)=g_{p}^{2}(0) / 12\left(\int f^{2}(x) d x\right)^{2}\left(\lambda_{p}(F)-\frac{1}{4}\right) .
\end{aligned}
$$

Now we shall evaluate the value of A.R.E. $\left(\hat{\theta}_{p} X\right)$. For this purpose we require following two lemmas.

Lemma 3. 1. Let $X_{i, 1}, X_{i, 2}, \cdots, X_{i, N}$ be independent random samples from the population with c.d.f. $F\left(x-\theta_{i}\right), i=1,2, \cdots, c$, and let

$$
U^{\left(i, i i_{2} \cdots i r\right)}=\frac{1}{\left(\begin{array}{c}
N \\
2
\end{array}\right)} \sum_{\substack{\alpha, \beta=1 \\
\alpha<\beta}}^{N} \varphi\left(Z_{i_{1} i_{2} \ldots i r}, \alpha, Z_{j_{1} j_{2} \ldots j r}, \beta\right),
$$

where $Z_{i_{1 i_{2} \ldots i r, \alpha}}=X_{i, \alpha+\cdots+} X_{i_{r},{ }_{\alpha}}$ and $\varphi\left(Z_{\alpha}, Z_{\beta}\right)=1$ if $Z_{\alpha}+Z_{\beta}>0$, =0 otherwise. Then the random vector with components $N^{1 / 2}\left(U^{\left(i_{1} \cdots i r\right)}-E_{0} U^{\left(i_{1} \cdots i r\right)}\right)$ has a normal distribution with mean $O$ and covariance matrix $\left.\left(4\left[\lambda_{2}{ }_{(i 1 \ldots i r)}^{(G r} j_{1} \cdots j_{r}\right)-\frac{1}{4}\right]\right)$, where

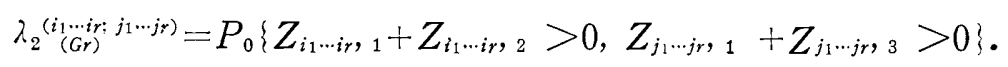

Proof is obuious from the general theory of generalized $U$-statistic (see 
[3] P. 964.).

Lemma 3. 2. For $\lambda_{p}(F)$ defined by (2.4) it holds that for all $F \in \widetilde{F}$

$$
\frac{1}{4} \leq \lambda_{2 m}(F) \leq \frac{3 m+1}{12 m}, m=1,2, \cdots .
$$

Proof The left inequality is easy from the Schwarz' inequality; $\lambda_{p}(F)$ $=\int f(x) G_{2 m-1}^{2}(x) d x \geq\left(\int f(x) G_{2 m-1}(x) d x\right)^{2}=\left(P_{0}\left\{X_{1}+\quad+X_{2 m}>0\right\}\right)^{2}=1 / 4$, for the distribution of $X_{1}, X_{2}, \cdots, X_{2 m}$ is symmetric about the origin. To prove the right inequality, consider the random vector $Y$ with components

$$
\begin{aligned}
& Y_{i_{11} i_{12} \cdots i_{1 m}}, Y_{i_{21} i_{22} \cdots i_{2} m}, \cdots, Y_{i_{m 1} i_{m 2} \cdots i_{m m}}, \\
& Y_{i_{11} i_{21} \cdots i_{m 1}}, Y_{i_{12} i_{22} \cdots i_{m 2}}, \cdots, Y_{j_{1 m} i_{2 m} \cdots i_{m m}},
\end{aligned}
$$

where $Y_{i_{1} i_{2} \cdots i_{m}}=N^{1 / 2}\left(U^{\left(i_{1} \cdots i_{m}\right)}-E_{0} U^{\left(i_{1} \cdots i_{m}\right)}\right)$ and $U^{\left(i_{1} \cdots i_{m}\right)}$ are defined in Lemma 3. 1. By (3. 3) the asymptotic covariance of $Y_{i_{1} \cdots i_{m}}$ and $Y_{j_{1} \cdots j_{m}}$ is given by

$$
\begin{aligned}
4\left[\lambda_{2}^{\left(i_{1} \cdots i_{m}: j_{1} \cdots j_{m}\right)}-\frac{1}{4}\right] & =0 \text {; if } i_{1}, \cdots, i_{m}, j_{1}, \cdots, j_{m} \text { are all different } \\
, & =\frac{1}{3} \text {; if }\left(i_{1} i_{2} \cdots i_{m}\right)=\left(j_{1} j_{2} \cdots j_{m}\right) \\
& =4\left(\lambda_{2 m}(F)-\frac{1}{4}\right) ; \text { otherwise. }
\end{aligned}
$$

Hence the asymptotic convariance matrix of $Y$, denoted by $\Sigma_{m}$, is written as follows.

(3. 6)

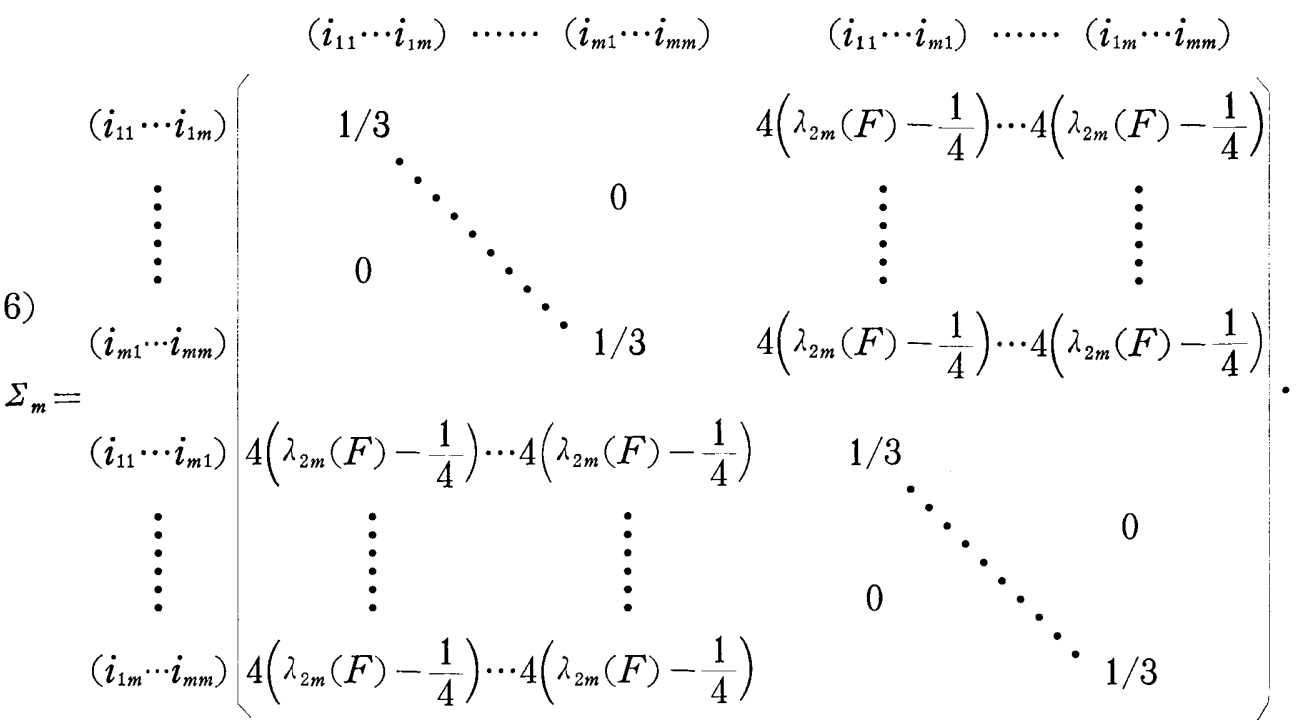

Put $\lambda_{2 m}(F)-1 / 4=r / 12$, then the determinant of $\Sigma_{m}$ is 


$$
\operatorname{det} \Sigma_{m}=\left(\frac{1}{3}\right)^{2 m}\left|\begin{array}{lll}
1 & 0 & r \cdots r \\
\ddots & \vdots & \vdots \\
0 & 1 & \cdots \\
r \cdots r & 1 & 0 \\
\vdots & \vdots & \ddots \\
r \cdots r & 0 & 1
\end{array}\right|=\left(\frac{1}{3}\right)^{2 m}\left(1-m^{2} r^{2}\right)
$$

Since det $\Sigma_{m} \geq 0$, we have $r \leq 1 / m$, which implies $\lambda_{2 m}(F) \leq(3 m+1) / 12 m$, as was to be proved.

We shall denote by $\mathfrak{F}^{*}$ the family of distributions which belong to $\mathfrak{F}$ and satisfy the condition of the theorem 2.1 .

Theorem 3. 1. Suppose that $p$ is even. Then

$$
\begin{aligned}
& \text { inf A.R.E. }\left(\hat{\theta}_{p} \mid X\right) \geq 0.864 \text {. } \\
& F \in \xi^{*}
\end{aligned}
$$

Proof We shall put $p=2 m, m=1,2, \cdots$, then

$$
\begin{aligned}
& g_{2 m}(0)=\int g_{m}^{2}(x) d x . \text { From }(3.1) \text { and lemma 3. 2., } \\
& \inf \text { A.R.E. }\left(\hat{\theta}_{2 m}(\bar{X})=i n f \frac{\sigma_{f}^{2} g_{2 m}^{2}(0)}{\lambda_{2 m}(F)-1 / 4}\right. \\
&=\inf \frac{12 \sigma_{g_{m}}^{2}\left(\int g_{m}^{2}(x) d x\right)^{2}}{12 m\left(\lambda_{2 m}(F)-1 / 4\right)} \geq \frac{\inf 12 \sigma_{g_{m}}^{2}\left(\int g_{m}^{2}(x) d x\right)^{2}}{\operatorname{sup~} 12 m\left(\lambda_{2 m}(F)-1 / 4\right)} \\
& \quad \geq \inf 12 \sigma_{g_{m}}^{2}\left(\int g_{m}^{2}(x) d x\right)^{2},
\end{aligned}
$$

where $\sigma_{g_{m}}^{2}$ is the variance of p.d.f. $g_{m}$. It has been shown by Hodges and Lehmann [1] that

$$
g_{m}(x)=\frac{3}{20 \sqrt{5}}\left(5-x^{2}\right) \text { if } x^{2} \leq 5,=0 \text { otherwise }
$$

attains the infimum value 0.864 of the last expression. This completes the proof.

Remark. For even $m$ there exists no underlying distribution $F(x)$ which satisfies (3. 8), since the characteristic function is

$$
(3 / 5 \sqrt{5})\left[\left(1 / t^{3}\right) \sin t \sqrt{5}-\left(\sqrt{5} / t^{2}\right) \cos t \sqrt{5}\right]
$$

which is negative for some $t$. The author presents a conjecture A.R.E. $\left(\hat{\theta}_{2 m} \bar{X}\right)>0.864$ for all $m>1$.

The above theorem does not hold for odd $p$, as is seen in Table II for $p=3$. In order to give an evaluation for odd $p$, we shall consider the random variable $Z_{t_{1 i_{2} \cdots i}, \alpha}, \alpha=1,2, \cdots, N$, given in lemma 3.1 and the statistic $U_{\left(i_{1} i_{2} \cdots i_{r}\right)}=N^{-1} \sum_{i=1}^{N} \psi\left(Z_{i_{1 i_{2} \ldots i r}, \alpha}\right)$, where $\psi(Z)=1$ if $Z>0,=0$ otherwise. A similar procedure as lemmas 3. 1 and 3. 2 will lead us to obtain 


$$
\frac{1}{4} \leq \lambda_{p}(F) \leq \frac{1+p}{4 p}, p=1,2, \cdots
$$

Though the upper bound of (3.9) is somewhat larger than that of (3. 4) for even $p$, it gives an evaluation of $\lambda_{p}(F)$ for odd $p$. Therefore we shall try to evaluate the value of A.R.E. $\left(\hat{\theta}_{p} X\right)$ for odd $p$ by means of (3. 9). Let $\widetilde{F}_{u}$ be the family of distributions which are unimodal and belong to $₹$. Then

Lemma 3. 3. ${ }^{(1)}$ If $F(x) \in \mathfrak{F}_{u}$, then $G_{p}(y) \in \mathfrak{F}_{u}$.

Proof It is sufficient to show that if $X$ and $Y$ are independent random variables with c.d.f. $F(x) \in \mathfrak{F}_{u}$ and $G(y) \in \mathfrak{F}_{u}$, respectively, then the c.d.f. $H(\boldsymbol{z})$ of the random variable $Z=X+Y$ belongs to $\mathfrak{F}_{u}$. Since $H(\boldsymbol{z}) \in \mathfrak{F}$ is obvious, we shall show the unimodality of $H(\boldsymbol{z})$. Let the p.d.f. of $F, G$ and $H$ be $f, g$ and $h$, respectively. Then for arbitrary $z_{2}>z_{1}>0$,

$$
\begin{aligned}
h\left(z_{2}\right) & -h\left(z_{1}\right)=\int_{-\infty}^{\infty}\left\{f\left(z_{2}-y\right)-f\left(z_{1}-y\right)\right\} g(y) d y \\
& =\int_{-\infty}^{\left(z_{1}+z_{2}\right) / 2}\left\{f\left(z_{2}-y\right)-f\left(z_{1}-y\right)\right\} g(y) d y+\int_{\left(z_{1}+z_{2}\right) / 2}^{\infty}\left\{f\left(z_{2}-y\right)-f\left(z_{1}-y\right)\right\} g(y) d y \\
& =\int_{\left(z_{1}+z_{2}\right) / 2}^{\infty}\left\{f\left(z_{2}-y\right)-f\left(z_{1}-y\right)\right\}\left\{g(y)-g\left(z_{1}+z_{2}-y\right)\right\} d y .
\end{aligned}
$$

Now $\left|z_{2}-y\right| \leq\left|z_{1}-y\right|$ and $y|\geq| z_{1}+z_{2}-y$ for $y \geq\left(z_{1}+z_{2}\right) / 2$, so that from symmetry and unimodality of $F, G$, it follows that $f\left(z_{2}-y\right) \geq f\left(z_{1}-y\right), g(y)$ $\leq g\left(z_{1}+z_{2}-y\right)$ for $y>\left(z_{1}+z_{2}\right) / 2$. Hence $h\left(z_{2}\right) \leq h\left(z_{1}\right)$, as was to be proved.

Let $\widetilde{F}_{u}^{*}$ be the family of distributions which are unimodal and belong to $\mathfrak{F}^{*}$. From lemma 3. $3 g_{2 m}(0) \geq g_{2 m-1}(x)$ for any $F \in \mathfrak{F}_{u}$. Therefore $g_{2 m}(0)=\int f(x) g_{2 m-1}(x) d x \leq g_{2 m-1}(0)$. Hence from theorem 3. 1,

$$
\begin{gathered}
\inf _{F \in \mho_{u^{*}} *} \sigma_{f}^{2} g_{2 m-1}^{2}(0) \geq \underset{F \in \mathfrak{F}_{u^{*}}}{\inf } \frac{\sigma_{g m}^{2}}{m} g_{2 m}^{2}(0) \\
\geq \frac{0.864}{12 m}, \text { for } m=1,2, \cdots
\end{gathered}
$$

Combining this with (3. 9), we obtain the theorem below.

Theorem 3. 2. For odd $p$ it holds that

$$
\inf _{F \in \mho_{i} *} A . R \cdot E \cdot\left(\hat{\theta}_{p}, \bar{X}\right) \geq 0.288 \frac{2 p}{p+1}
$$

Some numerical values of $g_{p}(0), \lambda_{p}(F)$ and A.R.E. $\left(\hat{\theta}_{p} \bar{X}\right)$ for normal, uniform and double exponential distributions are given in the following tables.

(1) The lemma and the proof was given in more generaliged form by professor K. Isii, Osaka University. 
Table I $f(x)=(1 / \sqrt{2 \pi}) \exp \left(-x^{2} / 2\right)$

\begin{tabular}{cccccc|cc}
\hline$p$ & 1 & 2 & 4 & 5 & 10 & 20 \\
\hline$g_{p}(0)$ & 0.3989 & 0.2829 & 0.1995 & 0.1784 & 0.1262 & 0.0892 \\
$\lambda_{p}(F)$ & 0.5000 & 0.3333 & 0.2902 & 0.2820 & 0.2659 & 0.2579 \\
A.R.E. $\left(\hat{\theta}_{p} \quad X\right)$ & 0.6366 & 0.9500 & 0.9894 & 0.9933 & 0.9983 & 0.9996 \\
\hline
\end{tabular}

Table II $f(x)=1 \quad x \in\left(-\frac{1}{2}, \frac{1}{2}\right),=0$ otherwise

\begin{tabular}{c|ccc|cc|c}
\hline$p$ & 1 & 2 & 3 & 4 & 5 & 6 \\
\hline$g_{p}(0)$ & 1.0000 & 1.0000 & 0.7500 & 0.6667 & 0.5990 & 0.5500 \\
$\lambda_{p}(\boldsymbol{F})$ & 0.5000 & 0.3333 & 0.3052 & 0.2909 & 0.2825 & 0.2771 \\
A.R.E. $\left(\hat{\theta}_{p} \mid \bar{X}\right)$ & 0.3333 & 1.0000 & 0.8490 & 0.9061 & 0.9192 & 0.9296 \\
\hline
\end{tabular}

Table III $f(x)=\frac{1}{2} e^{-x}$

\begin{tabular}{ccc|c|c|c|c}
\hline$p$ & 1 & 2 & 3 & 4 & 5 & 6 \\
\hline$g_{p}(0)$ & 0.5000 & 0.2500 & 0.1875 & 0.1563 & 0.1367 & 0.1230 \\
$\lambda_{p}(\boldsymbol{F})$ & 0.5000 & 0.3333 & 0.3032 & 0.2908 & 0.2809 & 0.2761 \\
A.R.E. $\left(\hat{\theta}_{p} \mid \boldsymbol{X}\right)$ & 2.0000 & 1.5000 & 1.3207 & 1.2439 & 1.2118 & 1.1582 \\
\hline
\end{tabular}

It would be interesting to compute the numerical values of A.R.E. $\left(\hat{\theta}_{p} \mid \bar{X}\right)$ with respect to the following distributions.

$$
\begin{gathered}
f(x)=\frac{\varepsilon}{\sqrt{2 \pi}} e^{-\frac{x}{2}}+\frac{(1-\varepsilon)}{2} e^{-x}, 0 \leq \varepsilon \leq 1 \\
f(x)=\frac{1}{\left(1+\frac{1+\alpha}{2}\right) 2^{1+(1+\alpha) / 2}} \exp \left\{-\frac{1}{2} x^{\frac{2}{1+\alpha}}\right\},-1<\alpha \leq 1 .
\end{gathered}
$$

These two families include a normal distribution $(\varepsilon=1, \alpha=0)$ as well as a double exponential distribution $(\varepsilon=0, \alpha=1)$. It is expected that for any $p=3,4, \cdots$ there exists a value of $\varepsilon$ or $\alpha$ for which A.R.E. $\left(\hat{\theta}_{p} \bar{X}\right)$ attains its maximum value $\geq 1$ at $p$.

\section{§4. Alternative estimators of $\theta$}

Suppose that $N$ observations $X_{1}, X_{2}, \cdots, X_{N}$ are divided in some way into $p$ groups, which denoted by $\left(X_{1}^{(1)}, \cdots, X_{n_{1}}^{(1)}\right),\left(X_{1}^{(2)}, \cdots, X_{n_{2}}^{(2)}\right), \cdots,\left(X_{1}^{(p)}, \cdots\right.$, $\left.X_{n_{p}}^{(p)}\right)$ where $n_{i}=\rho_{i} N, i=1,2, \cdots, p$ and $\rho_{1}+\rho_{2}+\cdots+\rho_{p}=1$. Then we can construct several alternative estimators of $\theta$ such as

(4. 1)

$$
\begin{aligned}
\hat{\theta}_{p}^{*} & =m e d \frac{X_{i_{1}}+X_{i_{2}}+\cdots+X_{i_{p}}}{p}, \\
i_{\alpha} & =1,2, \cdots, n_{\alpha} \\
\alpha & =1,2, \cdots, p
\end{aligned}
$$




$$
\begin{aligned}
\hat{\theta}_{p}^{* * *}=\frac{1}{p} \sum_{\alpha=1}^{p} \hat{\theta}^{(\alpha)}, \text { where } \hat{\theta}^{(\alpha)}=\operatorname{med}_{\substack{i<j\\
}} \frac{X_{i}^{(\alpha)}+X_{j}^{(\alpha)}}{2}, \\
i, j=1,2, \cdots, n_{\alpha}
\end{aligned}
$$

$$
\begin{aligned}
\hat{\theta}_{p}^{* * *}= & \operatorname{med}_{i>j} \frac{X_{i}+X_{j}}{2}, \text { where } X_{i}=\frac{1}{p} \sum_{\alpha=1}^{p} X_{i}^{(\alpha)} \\
& i, j=1,2, \cdots, n \\
& \text { provided } n_{1}=n_{2}=\cdots=n_{p}=n .
\end{aligned}
$$

\section{Theorem 4. 1 .}

(1) Under the same condition as in theorem 3. 1., $N^{1 / 2}\left(\hat{\theta}_{p}^{*}-\theta\right)$ has a limiting normal distribution with mean $O$ and variance $p^{-2}\left(\rho_{1}^{-1}+\cdots+\rho_{p}^{-1}\right)$ $\left(\lambda_{p}(F)-1 / 4\right) g_{p}^{-2}(0)$.

(2) Suppose that $G_{2}(y)$ has the derivative $g_{2}(0) \neq 0$ at $y=0$. Then $N^{1 / 2}\left(\hat{\theta}_{\hat{p}}^{* *}-\theta\right)$ has a limiting normal distribution with mean 0 and variance $p^{-2}\left(\rho_{1}^{-1}+\rho_{2}^{-1}+\cdots+\rho_{p}^{-1}\right)\left[12 g_{2}^{2}(0)\right]^{-1}$.

(3) Under the same condition as in (1) $N^{1 / 2}\left(\hat{\theta}_{*}^{* * *}-\theta\right)$ has a limiting normal distribution with mean 0 and variance $12\left[p g_{2 p}^{2}(0)\right]^{-1}$.

Proof (1) Since $\hat{\theta}_{p}^{*}$ can be represented by a $U$-statistic $T^{*}(X)=\left[\left(\begin{array}{c}n_{1} \\ 1\end{array}\right) \ldots\right.$ $\left.\left(\begin{array}{c}n_{p} \\ 1\end{array}\right)\right]^{-1} \neq\left\{\left(i_{1} \cdots i_{p}\right) ; X_{i_{1}}+X_{i_{2}}+\cdots+X_{i_{p}}>0, i_{\alpha}=1,2, \cdots, n ; \alpha=1,2, \cdots, p\right\}$ in the same way as (2.1), the proof is analogous to that of theorem3. 1. (2) follows from the relation $N^{1 / 2}\left(\hat{\theta}_{p}^{* *}-\theta\right)=p^{-1} \sum_{\alpha=1}^{p} \rho_{\alpha}^{-1 / 2} n_{\alpha}^{1 / 2}\left(\hat{\theta}^{(\alpha)}-\theta\right)$, where $n_{\alpha}^{1 / 2}\left(\hat{\theta}^{(\alpha)}\right.$ $-\theta), \quad \alpha=1,2, \cdots, p$, are independent and asymptotically normally distributed with mean 0 and variance $\left[12 g_{2}(0)^{2}\right]^{-1}$.

(3) $\lim _{N \rightarrow \infty} P_{\theta}\left\{N^{1 / 2}\left(\hat{\theta}_{p}^{* * *}-\theta\right) \leq u\right\}=\lim _{n \rightarrow \infty} P_{0}\left\{n^{1 / 2} \hat{\theta}_{p}^{* * *} \leq p^{-1 / 2} u\right\}$. Since $\bar{X}_{i}, i=1$, $2, \cdots, n$, are independent and identically distriduted with p.d.f. $p g_{p}(p x)$ when $\theta=0$, from the theorem 3.1. $n^{1 / 2} \hat{\theta}_{p}^{* * *}$ has a limiting normal distribution with mean 0 and variance $\left[12 p^{2} g_{2 p}^{2}(0)\right]^{-1}$, as was to be proved.

It is seen by the theorem that for $N$ fixed $n_{1}=n_{2}=\cdots=n_{p}$ is the best choice of the group sizes in order to make the asymptotic variance of $\hat{\theta}_{p}^{*}$ or $\hat{\theta}_{p}^{* *}$ minimum. In this case the estimator $\hat{\theta}_{p}^{*}$ has the same asymptotic distribution as $\hat{\theta}_{p}$. Now since $\hat{\theta}_{2}^{*}$ as well as $\hat{\theta}_{2}$ has the same asymptotic distribution as $\hat{\theta}_{p}^{* *}$, considering a trouble involved in computing $\hat{\theta}_{p}$ and $\hat{\theta}_{p}^{*}$, we might as well recomend $\hat{\theta}_{p}^{* *}$ as an estimator of $\theta$ when $N$ is large and $n_{1}=n_{2} \cdots=n_{p}$.

On the other hand for arbitrary $n_{1}, n_{2}, \cdots, n_{p}$ it will be preferable to use $\hat{\theta}_{p}, p=2 m, m=1,2, \cdots$, as an estimator of $\theta$, for $\hat{\theta}_{p}^{*}$ or $\hat{\theta}_{p}^{* *}$ has a large loss of efficiency in this case.

Since A.R.E. $\left(\hat{\theta}_{p}^{* * *} \bar{X}\right)=12 p \sigma_{f}^{2} g_{2 p}^{2}(0)=12 \sigma_{g_{p}}^{2}\left(\int g_{p}^{2}(x) d x\right)^{2}$, the infimum of A.R.E. $\left(\hat{\theta}_{\hat{p}}^{* * *} \bar{X}\right)$ never falls below 0.864 .

Therefore $\hat{\theta}_{p}^{* * *}$ will also be recommended for a practical use as an estimator 
of $\theta$ when sample size is large and $n_{1}=\cdots=n_{p}$.

\section{\$. Acknowledgement.}

The author should like to express his deepest gratitude to Prof. T. Kitagawa Kyûshû University, who suggested the problem and gave kind criticism and encouragement. The author also wishes his hearty thanks to Profs. M. Okamoto and K. Isii, Osaka University, for their generous help and guidance during the course of the entire work.

\section{References}

[1] Hodges, J.L.Jr. and Lehmann, E.L. (1956). The efficiency of some nonparametric competitors of the t-test. Ann. Math. Statist. 27. 324-355.

[2] Hodges, J.L.Jr. and Lehmann, E.L. (1963). Estimates of location based on rank test. Ann. Math. Statist. 34. 599-611.

[3] Lehmann, E.L. (1963). Robust estimation in Analysis of Variance. Ann. Math. Statist 34. 957-966. 